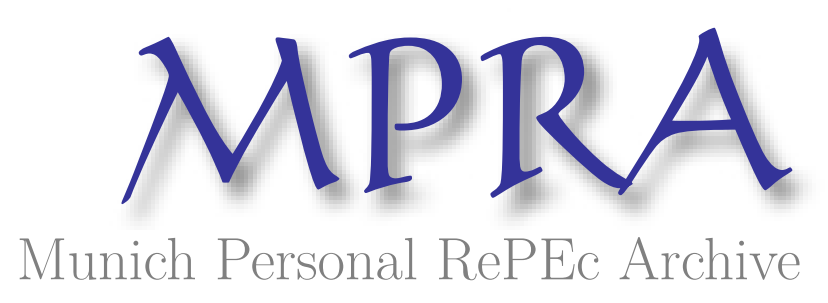

\title{
A "double coincidence" search model of money
}

Amendola, Nicola

19 March 2008

Online at https://mpra.ub.uni-muenchen.de/7839/

MPRA Paper No. 7839, posted 19 Mar 2008 15:02 UTC 


\title{
A "Double Coincidence" Search Model of Money *
}

\author{
Nicola Amendola ${ }^{\dagger}$ \\ University of Rome "Tor Vergata"
}

March 19, 2008

\begin{abstract}
According to Engineer and Shi $(1998,2001)$ and Berentsen and Rocheteau (2003), the double coincidence of wants problem seems to be not essential to rationalize the use of money in a search theoretic framework. This paper analyzes an endogenous price search model of money where there is universal double coincidence of wants. The existence of a monetary equilibrium depends, essentially, on the asymmetry in the role played by economic agents in the exchange and production processes. In particular, entrepreneurs are assumed to produce a fixed amount of a divisible consumption good by means of labour services provided by workers. Entrepreneurs can offer a co-operative (barter) contract or a monetary contract to workers. Under the co-operative contract real wages are determined in the labour exchange sector, while in the monetary regime real wages are determined in the commodity exchange sector. The monetary contract is proved to be an equilibrium strategy provided that: (i) the workers' labour disutility is sufficiently high and/or (ii) the entrepreneurs' bargaining power in the commodity market is sufficiently large relative to their bargaining power in the labour market. The rationale for money comes from the fact that entrepreneurs use it as an instrument to maximize their output share.
\end{abstract}

JEL code: D 78, E 40

Keywords: Money, Search, Double Coincidence, Bargaining

\section{Introduction}

The key feature used to motivate the existence of a monetary equilibrium, in the standard search theoretic analytical framework ${ }^{1}$ (Kyiotaki and Wright, 1991, 1993), is the the well known double coincidence of wants problem. According to the classical Jevons'

${ }^{*}$ I would like to thank all the participants to the European Workshop in Monetary Theory, held in the University of Rome Tor Vergata 27-28 october 2006, and in particular Aleksander Berentsen, Jean Cartelier, Marcello Messori, and Christopher Waller for their useful observations on a previous version of this paper. The usual disclaimers apply.

${ }^{\dagger}$ E-mail: nicola.amendola@uniroma2.it, Tel +39 (06) 72595730

${ }^{1}$ For an extensive survey on the search theoretic approach to the foundations of monetary theory see Rupert et al, 2000 and Shi, 2006 
argument, money emerges as an equilibrium transaction strategy because it allows to relax the double coincidence of wants requirement and, by this way, to reduce the mean waiting time to achieve the desired allocations.

More recently, however, some authors questioned that the double coincidence problem is essential to the existence of a monetary equilibrium. Engineer and Shi (1998) and Berentsen and Rocheteau (2003) prove that, in a search model with imperfectly transferable utility and demand asymmetries, the use of money can improve on the equilibrium allocations even if there is a universal double coincidence of wants. The asymmetry in bargaining across matches is at the core of Engineer and Shi (2001); money can be valued in equilibrium provided that it offers adequate bargaining terms relative to real assets.

The main aim of this paper is to show that the existence of a monetary equilibrium can be also obtained introducing asymmetries in the exchange and production structure. In particular, a search theoretic model with universal double coincidence of wants is analyzed where, in contrast to the prevailing literature, production can not be carried out by a single agent, but is modelled as an exchange relationship between entrepreneurs and workers. Entrepreneurs can produce a consumption goods by means of labour services provided by workers. Therefore, under the no auto-production assumption, a complementary relationship between entrepreneurs and workers emerges, togheter with a strong asymmetry in the role played by the two types of agents in the exchange and production process. Entrepreneurs supply consumption goods and demand labour services, while workers supply labour services and demand consumption goods.

Provided that there is no specialization on production and consumption and there are no real endowments at the beginning of the story, the main strategic problem faced by the entrepreneurs is how to carry out production. According to the first and more immediate solution, entrepreneurs and workers bargain over their respective output share and then realize production by means of workers' labour services and entrepreneurs' skills. However, there is another possible strategy available to the entrepreneurs: they can borrow from a bank a money unit which can be used to purchase labour services and to produce. After this stage, the entrepreneurs can go to an exchange sector where, according to a bargaining process, they can sell a fraction of their output against money, consume the residual fraction and pay back money to the bank. In other words, the entrepreneurs can decide to extend the transaction sequence contracting the real wage in the exchange sector instead of in the production sector.

Following the definition introduced by Keynes (1933), the first strategy characterizes a co-operative economy, while the second one can be easily interpreted as the exchange and production strategy prevailing in a monetary economy.

There are at least three differences between a co-operative and a monetary economy, which prove to be relevant for the monetary foundations' problem: (i) the bargaining process between workers and entrepreneurs takes place at different stages, (ii) the bargaining process between workers and entrepreneurs takes place in different sectors, (iii) the bargaining process involves different entrepreneurs and different workers.

According to point (i), in a co-operative economy entrepreneurs and workers bargain over the output shares before that production is actually realized while, in a monetary 
economy, the bargaining process takes place in a successive stage, namely in the exchange sector. As long as there is a positive labour disutility cost, the difference concerning the timing of the bargaining process has an interesting implication. As a matter of fact, in a monetary economy the entrepreneurs bargain with workers who are free from labour disutility costs. Because of that, in a monetary equilibrium the workers can be willing to accept a real wage lower than the real wage to be paid by an entrepreneur who decide to deviate, offering a co-operative arrangement. There is a labour disutility effect that could prevent potential deviations from the monetary equilibrium.

With reference to point (ii), we observe that in a co-operative economy entrepreneurs and workers bargain directly in the production sector. On the contrary, in a monetary economy agents face a longer transaction sequence, and real wages are determined in the exchange sector, where entrepreneurs and workers appears as sellers and buyers respectively. There are no general reasons to assume that the entrepreneurs' bargaining power in the exchange sector is equal to their corresponding bargaining power in the production sector. If the first one is sufficiently large relative to the second one, another argument in favour of the monetary equilibrium comes to the fore.

Regarding point (iii), it should be noted that, in a co-operative economy, production and exchange activities constitute a self-contained process; the same couple of entrepreneurs and workers produce the consumption good and consume it according to a previously defined sharing rule. On the opposite, in a monetary economy the production and exchange process is no more a self-contained process. Because of the decentralized search structure, a single worker bargains over quantities with an entrepreneur who is necessarily not the same entrepreneur who hired him in the previous period; the production couple is always different from the exchange couple. As a consequence, from the entrepreneurs' perspective, money is a device to avoid a commitment on quantities in the production sector whenever, because of point (i) and (ii), it would be better to bargain over quantities in the exchange sector.

Eventually, it should be emphasized that in our analysis the problem of the existence of a monetary equilibrium is logically separated from efficiency considerations. The rationale for money depends on what money can do for a specific class of agents, namely the entrepreneurs. The monetary economy is clearly inefficient, because it extends the transactions chain without producing any social advantage. On the other hand, monetary transactions could modify the surplus distribution in favour of the entrepreneurs. If the efficiency loss is more than compensated by the gains from surplus distribution, the entrepreneurs would prefer to produce in a monetary economy. Entrepreneurs may use money as an instrument to maximize their output share.

The rest of the paper is organized as follows. In Section 2 we introduce the basic model, where a positive labour disutility cost is assumed and the bargaining weights are constrained to be homogeneous across sectors and agents; this allows us to explore the implications of point (i). In order to analyze point (ii), Section 3 extends the previous model allowing for heterogeneous bargaining weights. Section 4 is dedicated to the efficiency property of the monetary equilibrium. Conclusions follow. 


\section{The Model}

The economy ${ }^{2}$ is populated by a continuum of agents of unitary mass and time is continuous and goes forever. Population consists of $E$ entrepreneurs and $1-E$ workers. Entrepreneurs can produce one unit of a divisible consumption good by means of labor services. Workers are not able to directly produce consumption goods, but they can provide labour services to the entrepreneurs. Therefore, there is a technological complementarity between entrepreneurs and workers, and production, as well as consumption, implies an exchange activity between the two types of agents. Labour services are indivisible and homogeneous across workers and entail a disutility cost $\delta<1$.

Entrepreneurs and workers preferences are represented by a linear utility function $u(Q)=Q$, where $Q$ is the fraction of the output consumed. Consumption is necessary in order to produce and to work again ${ }^{3}$ and there is no specialization on production and consumption. Hence, there is always a double coincidence of wants between entrepreneurs and workers and every possible explanation for the existence of money should lie elsewhere. We also assume that there are no real endowment at the beginning of the story.

With regard to the matching technology, we assume that agents meet pairwise according to a Poisson process with a constant parameter $\beta$ normalized, for the sake of notation, to one. However, in order to emphasize the role of the asymmetric exchange structure, we choose to minimize search frictions adopting an endogenous matching framework without commitment ${ }^{4}$. The endogenous matching rule can be conditioned on agent type while, within types, meetings are at random.

In the above framework, entrepreneurs can carry out production following two possible strategies: $(i)$ when an entrepreneur meets a worker he offer him the access to a bargaining process over the sharing rule of the consumption good. The outcome of this bargaining process is identified by the symmetric axiomatic Nash bargaining solution with zero treath point. After this stage, the worker provides labour services, production is realized and the two agents split the consumption good according to the previously defined bargaining solution; (ii) an entrepreneur can applies a bank for an indivisible unit of fiat money. Then, when he meets a worker, he can offer him money against labour services. If the worker accepts, the entrepreneur produces the consumption good and goes to an exchange sector where, according to a symmetric axiomatic Nash bargaining solution with zero treath point, he sells a fraction of his consumption good to a worker against money. At the end of this stage he can repay the bank and come back to the initial position.

Workers do not have access to the credit system, and the financing cost is equal to zero. Moreover we assume that the bank can impose an infinite default penalty, so that entrepreneurs have always a positive incentive to give back money.

We refer to strategy (i) as a co-operative contract, while strategy (ii) could be easily

\footnotetext{
${ }^{2}$ The structure of the model is largely borrowed from Amendola (2008).

${ }^{3}$ This implies that even if there is no phisical obstacle to storing consumption goods, agents cannot store consumption goods for more than a single exchange session.

${ }^{4}$ See Corbae et Al. (2002) and Corbae et Al. (2003)
} 
interpreted as a monetary contract. Entrepreneurs decide about the type of contract they will offer to workers, while workers can only decide to accept or reject the entrepreneurs' offer.

The symmetric axiomatic Nash bargaining solution is assumed because of its uniqueness and analytical tractability. However this solution can be always interpreted as the unique subgame perfect Nash equilibrium of a properly defined sequential bargaining game (Binmore, Rubinstein, and Wolinsky, 1986). For instance, the bargaining protocol behind the co-operative contract can be described as follows: at the first stage of the game, Nature randomly selects, with equal probability, one of the two players. The selected player can offer a sharing rule for the consumption good unit. If the other player accepts the game stops, if he refuses the game continues and, after a time interval $\Delta t$, Nature randomly selects, with the same probability distribution, a new player. Agents can exit at any stage of the game, but they can not meet other agents during $\Delta t$. It is possible to prove that the sequential game above described has a unique subgame perfect Nash equilibrium and that, at the equilibrium, the first proposal is always accepted. Moreover, as the recontracting time lag $\Delta t$ approaches to zero, the equilibrium outcome of the game approaches to the symmetric axiomatic Nash bargaining solution with zero treath point.

In the rest of the paper, we will focus only on steady state and pure strategy equilibria. Our main purpose is to prove that the monetary contract could be a sustainable equilibrium, i.e. if all entrepreneurs offer a monetary contract, no entrepreneur has incentives to offer a co-operative contract.

\subsection{Endogenous Matching and Steady State Distribution}

Let us assume that entrepreneurs decide to offer a monetary contract and that workers are willing to accept it. There are four possible states for our economy: entrepreneurs can be in the production sector or in the exchange sector; in the first case they are endowed with a money unit while, in the second case they are endowed with a consumption good unit. We refer to the first state as state $p=$ producer and to the second one as state $s=$ seller. Symmetrically, workers can be in the production sector, endowed with potential labour services, or in the exchange sector endowed with a money unit. We refer to these states respectively as state $w=$ active worker and state $b=$ buyer.

In an endogenous matching framework without commitment agents decide who want to meet according to an optimal matching rule. In the our specific setup the optimal endogenous matching rule is the following one: (a) active workers decide to meet producers an viceversa; (b) sellers decide to meet buyers an viceversa. Any other possible rule is sub-optimal entailing no exchange opportunities.

According to the previously defined matching rule, meeting probabilities are the following:

$$
\begin{aligned}
p_{s b} & =\min \left\{\frac{n_{b}}{n_{s}}, 1\right\} ; p_{b s}=\min \left\{\frac{n_{s}}{n_{b}}, 1\right\} \\
p_{p w} & =\min \left\{\frac{n_{w}}{n_{p}}, 1\right\} ; p_{w p}=\min \left\{\frac{n_{p}}{n_{w}}, 1\right\}
\end{aligned}
$$


where $n_{i} i=b, s, p, w$ denotes the agents' distribution among states and $p_{i j}$ is the probability that a type $i$ agent meets a type $j$ agent. Meeting probabilities are regulated by a short side rule.

We will restrict our attention to steady state equilibria and we assume $1-E \geq E$; the number of workers is equal or larger than the number of entrepreneurs. The meaning of this restriction is quite simple: we choose to analyze an economy in which there is a structural excess supply of labour or, at least, a zero excess supply.

According to the above meeting probabilities, steady state conditions are:

$$
\begin{gathered}
n_{b} \min \left\{\frac{n_{s}}{n_{b}}, 1\right\}=n_{w} \min \left\{\frac{n_{p}}{n_{w}}, 1\right\} \\
n_{b}+n_{w}=1-E \geq E=n_{p}+n_{s} \\
n_{b}=n_{s}
\end{gathered}
$$

Condition (3) comes from the fact that in the exchange sector there is always a flowin equilibrium and, as a consequence, there is always a stock equilibrium ${ }^{5}$. From (2) and (3) we have $n_{w} \geq n_{p}$. Hence, from (1) it follows $n_{s}=n_{p}$. The steady state distribution is:

$$
n_{s}=n_{p}=n_{b}=\frac{E}{2} ; n_{w}=\frac{2-3 E}{2}
$$

while

$$
p_{w p}=\min \left\{\frac{n_{p}}{n_{w}}, 1\right\}=\frac{E}{2-3 E}=\gamma(E)
$$

and

$$
p_{s b}=p_{b s}=p_{p w}=1
$$

Note that $1-E>E$ implies $\gamma(E)<1$; the probability that an active worker meets a producer is strictly less than one. On the contrary, if $1-E=E, \gamma(E)=1$ and there are no short sides in the production sector. With all the due caveats, we can take $\gamma(E)$ as a proxy for the unemployment rate, referring to a $\gamma(E)=1$ economy as to a full employment economy.

\subsection{Optimality Conditions}

Given the steady state distribution defined in (4), we can write down the following standard Bellmann equations for a monetary equilibrium:

$$
\begin{gathered}
r V_{p}=V_{s}-V_{p} \\
r V_{s}=V_{p}-V_{s}+Q_{m}
\end{gathered}
$$

\footnotetext{
${ }^{5}$ Producers go to the exchange sector if and only if they meet an active worker and viceversa. This implies that there is always a flow-in equilibrium between producers and active worker, as for every producers coming into the exchage sector there is always a corresponding active worker coming into the same sector. However, if the above condition holds true, the only possible steady state distribution consistent with the initial condition $n_{s}^{0}=n_{b}^{0}=0$ (no real endowments assumption) requires $n_{s}=n_{b}$.
} 


$$
\begin{aligned}
r V_{w} & =\gamma(E)\left[V_{b}-V_{w}-\delta\right] \\
r V_{b} & =V_{w}-V_{b}+1-Q_{m}
\end{aligned}
$$

where $V_{j} ; j=s, b, w, p$ is the value function of a state $j$ agent, $r>0$ is the intertemporal rate of preference, $\delta$ is the working disutility and $1-Q_{m}$ is the quantity of the consumption good exchanged against one unit of money. Momentarily, we take $Q_{m} \in[0,1]$ as an exogenous value; we will determine the equilibrium value of $Q_{m}$ in the next section.

The interpretation of conditions (5)-(8) is the usual one. The flow return of state $j$ must be equal to the gain from trade in state $j$. For instance, the flow return of an active worker must be equal to the probability $\gamma(E)$ with which he meets a producer times the gain from subscribing a monetary contract, where this last one is given by the value function of becoming a buyer $V_{b}$ minus the value function of being an active worker $V_{w}$ minus the working disutility $\delta$.

However, because of the non-negativity constraint on the value functions, not all the possible values of $Q_{m}$ are consistent with the optimality conditions. From equations (5)-(8) we derive the following producers' participation constraints:

$$
\begin{gathered}
r V_{p} \geq 0 \\
V_{p}-V_{s}+Q_{m} \geq 0
\end{gathered}
$$

Resorting once again to the optimality conditions, we obtain:

$$
r V_{p}=\frac{Q_{m}}{r+2} \geq 0 \text { for every } Q_{m} \in[0,1]
$$

and

$$
V_{p}-V_{s}+Q_{m}=\frac{Q_{m}(r+1)}{r+2} \geq 0 \text { for every } Q_{m} \in[0,1]
$$

Hence, participation constraints (9) and (10) are always satisfied. The same is not true with reference to the workers' participation constraints which are the following ones:

$$
\begin{gathered}
V_{b}-V_{w}-\delta \geq 0 \\
V_{w}-V_{b}+1-Q_{m} \geq 0
\end{gathered}
$$

Remembering the optimality conditions, we can write the two previous constraints in the following form:

$$
\begin{gathered}
Q_{m} \leq 1-\delta(1+r)=\bar{Q}_{m} \\
Q_{m} \leq 1-\delta \frac{\gamma}{r+\gamma}=\widetilde{Q}_{m}
\end{gathered}
$$

First of all we observe that $\widetilde{Q}_{m}>\bar{Q}_{m}$. This means that given constraint (13), constraint (14) is never binding. However, we need also $\bar{Q}_{m} \geq 0$ and this last condition is satisfied if and only if:

$$
\delta \leq \frac{1}{r+1}
$$


We conclude that if the constraint (15) holds true, a feasible monetary solution $Q_{m}$ must belong to the subset $\left[0, \bar{Q}_{m}\right]$, where $1-\bar{Q}_{m}$ can be interpreted as a sort of reservation wage. It should be noted that the reservation wage is increasing in $\delta$ and $r$, where this property has a quite obvious interpretation. If the working disutility increases there must be a corresponding increase in the reservation wage. If $r$ increases, the cost of delaying consumption increases and this cost must be compensated by a higher reservation wage.

\subsection{Price Theory}

The value $Q_{m}$ is a monetary price and must be determined endogenously as a result of a bargaining process between buyers and sellers. Following Trejos and Wright (1995), we assume that this bargaining process leads to an axiomatic rational expectation Nash bargaining solution (reNBS) with zero threat point. In order to characterize the reNBS solution we follow a two step procedure. First of all we identify the equilibrium quantity $q_{m}$ determined in a single match. This quantity solves the following problem:

$$
\begin{gathered}
\max _{q_{m}}\left(V_{p}+q_{m}\right)\left(V_{w}+1-q_{m}\right) \\
\text { s.t. } V_{w}-V_{b}+1-q_{m} \geq 0
\end{gathered}
$$

where $V_{p}=V_{p}\left(Q_{m}\right), V_{w}=V_{w}\left(Q_{m}\right), V_{b}=V_{b}\left(Q_{m}\right)$ and $Q_{m}$ is the quantity that the single agent expects to obtain on the market. The solution of problem (16) determines an optimal reply function $q_{m}=f\left(Q_{m}\right)$. However, under the rational expectation hypothesis $Q_{m}=q_{m}$, so that a fixed point for $f\left(Q_{m}\right)$ identifies the reNBS $Q_{m}^{*}$.

We define an admissible monetary equilibrium a list $\left(V_{i}, i=p, w, b, s ; Q_{m}^{*}>0\right)$ satisfying the following conditions: (i) $q_{m}=Q_{m}=Q_{m}^{*}$ solves the bargaining problem (16) taking $V_{p}=V_{p}\left(Q_{m}\right), V_{w}=V_{w}\left(Q_{m}\right)$ and $V_{b}=V_{b}\left(Q_{m}\right)$ as given, (ii) $V_{p}, V_{w}, V_{b}$ and $V_{s}$ solves the optimality conditions (5)-(8) under constraint (13), taking $Q_{m}$ as given.

The following proposition holds true:

Proposition 1 An admissible monetary equilibrium exists if and only if $\delta \leq 1 /(1+r)$. If $\delta \leq \alpha(r)$, where $\alpha(r)$ is a decreasing function of $r$, the reNBS $Q_{m}^{*}$ is unique and solves the unconstrained version of problem (16). If $\alpha(r)<\delta \leq 1 /(1+r)$ the inequality constraint in (16) is binding and $Q_{m}^{*}=\bar{Q}_{m}$.

Proof. First of all we observe that an admissible monetary solution $Q_{m}^{*}$ must belong to $\left[0, \bar{Q}_{m}\right]$, where this last subset is a non-empty set if and only if $\delta \leq 1 /(1+r)$. Let us suppose now that the inequality constraint in problem (16) is not binding. From the first order condition for the unconstrained version of (16) we derive:

$$
q_{m}=\frac{1}{2}+\frac{V_{w}\left(Q_{m}\right)-V_{p}\left(Q_{m}\right)}{2}=f\left(Q_{m}\right)
$$

where, from optimality conditions (5)-(8), we have:

$$
V_{p}=\frac{Q_{m}}{r(r+2)} ; V_{w}=\frac{\gamma\left[1-Q_{m}-(r+1) \delta\right]}{r(r+1+\gamma)}
$$


It is immediate to prove that $f$ is a continuous differentiable function with $\partial f / \partial Q_{m}<0$. Therefore, we can rule out multiple solutions. However, we still have to prove the existence of an admissible unconstrained solution. From (17) we have $f(0)>0$; as a consequence, a necessary and sufficient condition for the existence of an unconstrained solution is $f\left(\bar{Q}_{m}\right) \leq \bar{Q}_{m}$. This implies, as a matter of fact, that the decreasing curve $f\left(Q_{m}\right)$ intersects the $45^{0}$ line at some point between 0 and $\bar{Q}_{m}$, i.e. that an unconstrained solution to $f(Q)=Q$ exists. Note that if $Q_{m}=\bar{Q}_{m}$ we have $V_{w}=0$ and, from (17), we obtain:

$$
q_{m}=\frac{1}{2}\left(1-\frac{\bar{Q}_{m}}{r(r+2)}\right)
$$

Hence, an admissible unconstrained reNBS exists if and only if:

$$
\frac{1}{2}\left(1-\frac{\bar{Q}_{m}}{r(r+2)}\right) \leq \bar{Q}_{m} \Leftrightarrow \delta \leq \frac{(r+1)}{2(r+1)^{2}-1}=\alpha(r)
$$

where $\alpha(r)$ is a decreasing function of $r$. On the contrary, if $\delta>\alpha(r)$ the function $f(Q)$ never intersects the $45^{0}$ line before $\bar{Q}_{m}$ and the constraint of problem (16) becomes binding. In this last case, the reNBS $Q_{m}^{*}$ is simply determined by the constraint, i.e. $Q_{m}^{*}=\bar{Q}_{m}$.

We conclude that if, for a given intertemporal preference rate $r$, the working disutility $\delta$ is not too high or, for a given labour disutility $\delta$, agents are sufficiently patient $(r$ is not too high) an admissible monetary equilibrium always exists. The interpretation of Proposition 1 is quite intuitive. In a monetary economy nor producers neither active workers can consume output immediately. They have to go to the exchange sector as a sellers and as a buyers respectively. In particular, with reference to active workers, the cost of delaying consumption depends on $r$, and if this cost is too high, relative to their disutility $\delta$, the monetary exchange system may not be viable.

\subsection{Existence of a Monetary Equilibrium}

In the previous sections we assumed that producers always decide to offer a monetary contract. In other words, we assumed the monetary equilibrium and we proved that this equilibrium can be consistent with the individuals' rationality constraints. In order to prove the existence of the monetary equilibrium we have to verify that there is no room for a deviating contract. More specifically, we have to characterize the conditions, if any, under which, in a monetary economy, no entrepreneur has incentive to offer a co-operative contract instead of a monetary contract.

Before analyzing the existence problem it will be useful to introduce the following result:

Proposition 2 A permanent deviation from the monetary contract is an optimal strategy if and only if a single deviation from the monetary contract is profitable.

Proof. The instantaneous pay-off of a producer is bounded below, therefore we can apply the unimprovability criterion of dynamic programming. Suppose that a single 
deviation form a monetary contract is profitable. As a consequence, the strategy that prescribe to do not deviate from the monetary contract is improvable in a single step. However, given the stationarity of the value function $V_{p}$, this implies that any finite deviation strategy is improvable in a single step and the only unimprovable, i.e. optimal strategy, is to permanently deviate from the monetary contract. Suppose, now, that a permanent deviation strategy is optimal. This implies that the monetary strategy is improvable in a single step, i.e. that a single deviation from the monetary contract is always profitable.

There are two main implications of Proposition 2. The first one is that if a producer decides to deviate from the monetary contract then he decides to deviate permanently; as a consequence we can restrict our attention to the permanent deviation strategy. The second one is that in order to check for the profitability of a permanent deviation strategy we can simply check the profitability of a single deviation strategy.

Let us define $q_{c}$ as the quantity traded in a single co-operative arrangement. A deviating producer obtains the pay-off $V_{p}^{c}\left(Q_{c}\right)+q_{c}$, where $V_{p}^{c}=Q_{c} / r$ denotes the value function of a producer who decides to offer always a co-operative contract and $Q_{c}$ is the quantity he expects to obtain under a co-operative arrangement. The corresponding active worker pay-off is $V_{w}\left(Q_{m}^{*}\right)+1-q_{c}-\delta$. The value $q_{c}$ is endogenously determined as a solution of the following optimization problem:

$$
\begin{gathered}
\max _{q_{c}}\left(V_{p}^{c}+q_{c}\right)\left(V_{w}+1-q_{c}-\delta\right) \\
\text { s.t. } q_{c}<1-\delta
\end{gathered}
$$

First order condition for the unconstrained problem (20) leads to the following best reply function:

$$
q_{c}=\frac{1}{2}+\frac{V_{w}-V_{p}^{c}}{2}-\frac{\delta}{2}=g\left(Q_{c}, Q_{m}^{*}\right)
$$

The best reply function depends on the equilibrium value $Q_{m}^{*}$ and on $Q_{c}$. As before, rational expectation assumption implies $q_{c}=Q_{c}=Q_{c}^{*}$. However, differently from problem (16), we can now prove that the reNBS for problem (20) is always unconstrained.

Lemma 1 For every $Q_{m}^{*}$ there is always a unique value $0<Q_{c}^{*}<1-\delta$ such that $Q_{c}^{*}=g\left(Q_{c}^{*}, Q_{m}^{*}\right)$

Proof. First of all, we observe that $g$ is a continuous differentiable function, and $\partial g / \partial Q_{c}<0$. Moreover $g\left(0, Q_{m}^{*}\right)=(1 / 2)\left[V_{w}+1-\delta\right]>0$ and, remembering equations (18), we have $g\left(1-\delta, Q_{m}^{*}\right)<1-\delta$. This means that a unique value $0<Q_{c}^{*}<1-\delta$ always exists such that $Q_{c}^{*}=g\left(Q_{c}^{*}, Q_{m}^{*}\right)$.

It is also interesting to note that $g\left(Q_{m}^{*}, Q_{m}^{*}\right)<f\left(Q_{m}^{*}\right)=Q_{m}^{*}$ and this implies that $Q_{c}^{*}<Q_{m}^{*}$. At the equilibrium, the deviating co-operative contract returns a quantity lower than the quantity obtained in a monetary contract. This is because the two contractual problems take place at different stages; there is a different matching sequence involving different subjects. In a deviating co-operative contract the producer bargain 
with an active worker who is suffering a disutility $\delta$. In a monetary contract the seller bargains with a buyer who has already paid the disutility $\operatorname{cost} \delta$. As a consequence, the reservation pay-off of an active worker is higher than the reservation pay-off of a buyer.

However, the inequality $Q_{c}^{*}<Q_{m}^{*}$ does not imply, by itself, that a deviation from the monetary contract is not profitable. In particular, we can state that a deviation from the monetary contract will be not profitable if and only if:

$$
V_{p}^{c} \leq V_{p}
$$

Where, from the optimality conditions (5)-(8), the above inequality can be written as:

$$
\frac{Q_{m}^{*}}{r+2} \geq Q_{c}^{*}
$$

We define a sustainable monetary equilibrium as an admissible monetary equilibrium such that condition (22) is always satisfied.

Proposition 3 Consider an unconstrained admissible monetary equilibrium. Hence, a sustainable monetary equilibrium always exists if and only if $\delta \in \mathcal{A}(\gamma, r) \subset \mathbb{R}_{+}$where $\mathcal{A}(\gamma, r)=\{\delta ; \varphi(r, \gamma) \leq \delta \leq \alpha(r)\}$ and $\varphi(r, \gamma)$ is an increasing function of $r$.

Proof. From Proposition 1, we know that a monetary equilibrium is admissible and unconstrained if and only if $\delta \leq \alpha(r)$. Let us consider now a permanent deviation from the monetary contract. From Proposition 2 we know that a permanent deviation is profitable if and only if a single deviation is profitable. Denote $Q_{c}^{*}(1)$ as the equilibrium quantity obtained under a single deviation strategy. From (17) and (21) we have

$$
Q_{c}^{*}(1)=Q_{m}^{*}-\frac{\delta}{2}
$$

This implies that the deviation is profitable if and only if

$$
Q_{m}^{*}-\frac{\delta}{2}>\frac{Q_{m}^{*}}{r+2}
$$

We conclude that a profitable deviation from the monetary contract does not exist if and only if

$$
Q_{m}^{*}-\frac{\delta}{2} \leq \frac{Q_{m}^{*}}{r+2} \Leftrightarrow Q_{m}^{*} \leq \frac{\delta}{2}\left(\frac{r+2}{r+1}\right)
$$

From equation (17) we can calculate the value $Q_{m}^{*}$ as a function of the parameters $r, \delta$ and $\gamma$

$$
Q_{m}^{*}=\frac{(r+2)[r+\gamma(1-\delta)]}{\left[\left(2(r+1)^{2}-1\right)+\gamma(2(r+1)+1)\right]}
$$

Substituting on we obtain:

$$
\delta \geq \frac{2(r+1)(r+\gamma)}{2(r+1)^{2}-1+\gamma[4(r+1)+1]}=\varphi(r, \gamma)
$$

and it is simply a matter of algebra to verify that $\partial \varphi(r, \gamma) / \partial r>0$. 
Proposition 4 Consider a constrained admissible equilibrium. Hence, a sustainable monetary equilibrium always exists if and only if $\delta \in \mathcal{B}(r) \subset \mathbb{R}^{+}$, where $\mathcal{B}(r)=$ $\{\delta ; \alpha(r)<\delta \leq \phi(r)\}$ and $\phi(r)$ is an increasing function of $r$.

Proof. From Proposition 1, we know that a monetary equilibrium is admissible and constrained if and only if $\alpha(r)<\delta \leq 1 /(1+r)$. Moreover at the constrained equilibrium we have $Q_{m}^{*}=\bar{Q}_{m}$. Hence, the equilibrium quantity obtained by a single deviating producer is

$$
Q_{c}^{*}(1)=\frac{1}{2}(1-\delta)-\frac{\bar{Q}_{m}}{2 r(r+2)}
$$

and the sustainability condition becomes

$$
\frac{\bar{Q}_{m}}{r+2} \geq \frac{1}{2}(1-\delta)-\frac{\bar{Q}_{m}}{2 r(r+2)} \Leftrightarrow \bar{Q}_{m} \geq \frac{r(r+2)(1-\delta)}{2 r+1}
$$

Remembering that $\bar{Q}_{m}=1-\delta(1+r)$, the previous one can be rewritten in the following way

$$
\delta \leq \frac{-\left(r^{2}-1\right)}{r(r+1)+1}=\phi(r)
$$

where $\partial \phi / \partial r<0$ and $\phi(r)<1 /(1+r)$ for every $r$.

Proposition 5 A sustainable monetary equilibrium always exists if and only if $\delta \in$ $\mathcal{A}(\gamma, r) \cup \mathcal{B}(r) \subset \mathbb{R}^{+}$.

Proof. It follows immediately from Propositions 3 and 4.

Proposition 5 characterizes the monetary equilibrium. In particular, it states that, for a given $r$ and $\gamma$, the working disutility $\delta$ must be neither too high nor too low. If $\delta$ is too high the active workers' participation constraint could be violated and the monetary equilibrium would be not admissible; if $\delta$ is too low producers can not take sufficient advantage from the monetary contract and the monetary equilibrium, even if admissible, is not sustainable. However, we can not be sure that such a $\delta$ effectively exists. In order to prove that we have to prove that $\mathcal{A}(\gamma, r)$ and $\mathcal{B}(r)$ are non-empty sets.

Proposition $6 \mathcal{A}(\gamma, r)$ is a non-empty set if and only if $r \leq 1 / 2$. Moreover, if $\gamma^{\prime}<\gamma$, $\mathcal{A}(\gamma, r) \subset \mathcal{A}\left(\gamma^{\prime}, r\right)$

Proof. Let us consider the particular case $\gamma=1$. Then:

$$
\varphi(r, 1)=\frac{r+1}{r+3}
$$

It is quite easy to verify that $\varphi(r, 1)$ has the following properties:

$$
\frac{\partial \varphi(r, 1)}{\partial r}>0 ; \varphi(0,1)=\frac{1}{2}<1=\alpha(0) ; \lim _{r \rightarrow \infty} \varphi(r, 1)=1>\lim _{r \rightarrow \infty} \alpha(r)=0
$$


As a consequence, a single value $r^{*}$ always exists such that if $r<r^{*}, \varphi(r, 1)<\alpha(r)$; if $r>r^{*}, \varphi(r, 1)>\alpha(r)$ and if $r=r^{*}, \varphi(r, 1)=\alpha(r)$. In particular, we find

$$
\varphi(r, 1)=\alpha(r) \Leftrightarrow r=\frac{1}{2}=r^{*}
$$

Therefore, if $r \leq 1 / 2, \varphi(r, 1)<\alpha(r)$ and $\mathcal{A}(1, r)$ is a non-empty set. Let us now define the following function:

$$
H(\gamma, r)=\varphi(r, 1)-\varphi(r, \gamma)
$$

From (25) we obtain:

$$
H(\gamma, r)=\frac{(r+1)(1-\gamma)(1-2 r)}{(r+3)\left\{2(r+1)^{2}-1+\gamma[4(r+1)+1]\right\}}
$$

and we can easily verify that the function $H(\gamma)$ has the two following properties:

$$
H(\gamma, r) \geq 0 \Leftrightarrow r \leq \frac{1}{2} \text { and } \gamma \in(0,1) ; \frac{\partial H(\gamma, r)}{\partial \gamma}<0
$$

this implies that if $r \leq 1 / 2$ the set $\mathcal{A}(\gamma, r)$ is non-empty and that for $\gamma^{\prime}<\gamma$, we have $\mathcal{A}\left(\gamma^{\prime}, r\right) \supset \mathcal{A}(\gamma, r)$.

Proposition $7 \mathcal{B}(r)$ is a non-empty set if and only if $r \leq 1 / 2$.

Proof. $\mathcal{B}(r)$ is non-empty if and only if $\phi \geq \alpha(r)$. However, the condition $\phi \geq \alpha(r)$ is always satisfied if and only if $r \leq 1 / 2$

Figure 1 illustrates how the set $\mathcal{A}(\gamma, r) \cup \mathcal{B}(r)$ varies with $\gamma$ and $r$. The dotted area identifies the parameters set consistent with unconstrained admissible monetary equilibria while the dashed area describes the set of costrained monetary equilibria. As $\gamma$ increases, the set associated to unconstrained equilibria enlarges, while the set of constrained equilibria does non depend on $\gamma$. The intuition is as follows: when $\gamma$ approaches to one the economy moves towards a full employment status. This implies that $V_{w}$ increases and, because of that, the deviating producer is able to extract more surplus form the workers. As a consequence, the monetary equilibrium becomes less credible. 


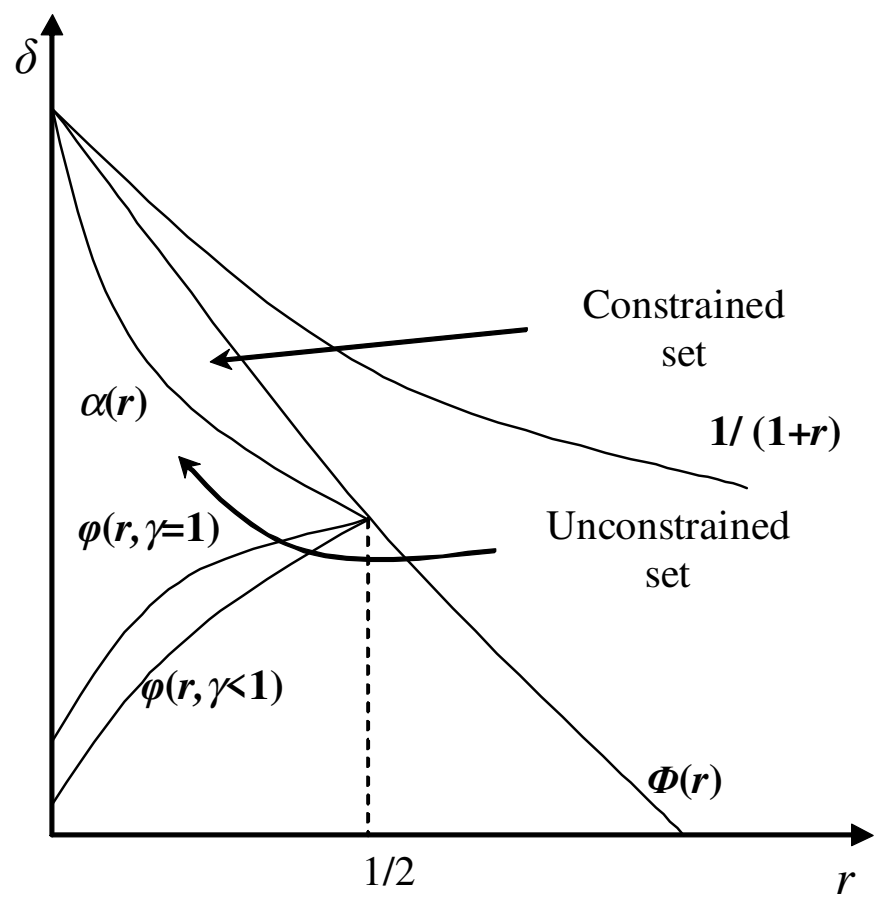

Figure 1: the set $\mathcal{A}(\gamma, r) \cup \mathcal{B}(r)$

A final remark, in this section, concern the behavior of $Q_{m}^{*}$ with respect to $\gamma$. It is quite easy to verify that, if $r \leq 1 / 2$, i.e. if a sustainable monetary equilibrium exists, we have $\partial Q_{m}^{*} / \partial \gamma>0$ (see equation (24)). This implies that the monetary price, given by $1 /\left(1-Q_{m}^{*}\right)$, increases as the economy moves towards a full employment status. In this sense we obtain a procyclical monetary price behavior which can be rationalized as follows. As $\gamma$ increases $V_{w}$ also increases; active workers, because of the unemployment rate reduction, are in a state better than before. At the same time this improvement weakens the workers' bargaining position in the commodity market and producers can take advantage of this obtaining an higher monetary price.

\section{The Generalized Economy}

In the above economy, the existence of a monetary equilibrium crucially depends on the introduction of a positive labour disutility $\operatorname{cost} \delta$. If $\delta$ were equal to zero, or even if it were too small, the monetary equilibrium would collapse. The reason is quite simple: the sustainability of a monetary equilibrium depends on the fact that in a monetary 
contractual arrangement the bargaining process takes place at a different stage with respect to a co-operative contractual arrangement. In particular, in a monetary economy entrepreneurs and workers bargain after the production stage, that is in a stage where workers are free from the labour disutility $\operatorname{cost} \delta$. Obviously, if $\delta=0$ this difference ceases to be relevant.

However, a monetary and a co-operative economy differ not only because agents bargain at different stage, but also because they bargain in different sectors. In the previous model we assumed that the bargaining power of a producer is equal to the bargaining power of a seller and this prevents us to analyze the consequences of this last kind of heterogeneity. If we want to appreciate this point we have to generalize the model allowing for heterogeneous bargaining powers across sectors. Therefore, in this section we extend the analysis introducing, in an otherwise unaltered environment, a generalized Nash bargaining solution concept. In order to simplify the algebra, we restrict our attention to a full employment economy, i.e. we set $\gamma=1$.

With reference to the monetary contract, the quantity exchanged in a single match solves now the following problem:

$$
\begin{gathered}
\max _{q_{m}}\left(V_{p}+q_{m}\right)^{\sigma}\left(V_{w}+1-q_{m}\right)^{1-\sigma} \\
\text { s.t. } V_{w}-V_{b}+1-q_{m} \geq 0
\end{gathered}
$$

where $0 \leq \sigma \leq 1$ is the seller's bargaining power, which can now be different from $1 / 2$.

The first order condition for the unconstrained problem (26) leads to the following generalized best reply function.

$$
q_{m}=\sigma+\sigma V_{w}-(1-\sigma) V_{p}=F\left(Q_{m}\right)
$$

Note that if, as in the previous section, $\sigma=1 / 2,(27)$ is equivalent to (17).

Resorting to equations (18) with $\gamma=1$, we can rewrite (27) in the following form:

$$
q_{m}(\sigma)=\sigma+\sigma\left[\frac{1-Q_{m}-(r+1) \delta}{r(r+2)}\right]-(1-\sigma)\left[\frac{Q_{m}}{r(r+2)}\right]=F\left(Q_{m}\right)
$$

As before, a fixed point for the best reply function identifies the generalized rational expectation bargaining solution (reGNBS) $Q_{m}^{*}(\sigma)$. We can now generalize Proposition 1 as follows.

Proposition 8 An admissible monetary equilibrium for the generalized economy exists if and only if $\delta \leq 1 /(1+r)$. If $\sigma \leq \bar{\sigma}(r, \delta)$, where $\bar{\sigma}$ is a decreasing function of $\delta$, the reGNBS $Q_{m}^{*}(\sigma)$ is unique and solves the unconstrained version of problem (26). If $\sigma>\bar{\sigma}(r, \delta)$ the inequality constraint of problem (26) is binding and $Q_{m}^{*}(\sigma)=\bar{Q}_{m}$

Proof. We will follow the same procedure adopted in Proposition 1. Let us assume that the constraint in problem $(26)$ is never binding. It is quite easy to verify that $\partial F / \partial Q_{m}<0$ and $F(0)>0$. In order to prove the existence of a unique unconstrained 
reGNBS $Q_{m}^{*}(\sigma)$ we have to prove that $F\left(\bar{Q}_{m}\right) \leq \bar{Q}_{m}$. Remember that if $Q_{m}=\bar{Q}_{m}$, $V_{w}=0$. As a consequence

$$
F\left(\bar{Q}_{m}\right)=\sigma-(1-\sigma)\left[\frac{\bar{Q}_{m}}{r(r+2)}\right]
$$

and $F\left(\bar{Q}_{m}\right) \leq \bar{Q}_{m}$ if and only if

$$
\sigma \leq \frac{(1+r)[1-\delta(1+r)]}{1+r-\delta}=\bar{\sigma}(r, \delta)
$$

where $\partial \bar{\sigma} / \partial \delta<0$. On the contrary, if $\sigma>\bar{\sigma}(r, \delta)$ there is no value $Q_{m}$ such that $F\left(\bar{Q}_{m}\right) \leq \bar{Q}_{m}$ (the function $F\left(Q_{m}\right)$ never intersects the $45^{0}$ line at $\left.Q_{m} \leq \bar{Q}_{m}\right)$. The inequality constraint of problem (26) is always binding and $Q_{m}^{*}(\sigma)=\bar{Q}_{m}$. However, because of the non-negativity constraint on $\bar{Q}_{m}$, we also need $\delta \leq 1 /(1+r)$.

The introduction of a generalized bargaining process weakens the constraint on labour disutility $\delta$. Even if $\delta>\alpha(r)$ we are now still able to identify an unconstrained admissible monetary equilibrium; the increased labour disutility can be compensated by a decreased seller's bargaining power.

At the same time, we have a new constraint on $\sigma$. If the seller's bargaining power is too high, the workers' participation constraint can be violated and this last ones have no incentive to subscribe the monetary contract. It is interesting to note that if $\delta=0$, $\bar{\sigma}=1$ and this means that there is no constraint on seller's bargaining power. In other words, if the labour disutility is equal to zero, the constraint of problem (26) will be never binding.

To conclude this section, we can compute the equilibrium value $Q_{m}^{*}(\sigma)$ which is given by

$$
Q_{m}^{*}(\sigma)=\frac{\sigma(r+1-\delta)}{(r+1)}=2 \sigma Q_{m}^{*}
$$

As expected, $Q_{m}^{*}(\sigma)$ is an increasing function of $\sigma$ and if $\sigma>1 / 2, Q_{m}^{*}(\sigma)>Q_{m}^{*}$.

\subsection{Monetary equilibrium}

As in section 1, in order to prove the existence of a sustainable monetary equilibrium we have to verify that a deviation from the monetary contract is not profitable. In the generalized setting the quantity $q_{c}$ contracted in a single match by a deviating producer solves the following problem:

$$
\begin{gathered}
\max _{q_{c}}\left(V_{p}^{d}+q_{c}\right)^{\eta}\left(V_{w}+1-q_{c}-\delta\right)^{1-\eta} \\
\text { s.t. } q_{c}<1-\delta
\end{gathered}
$$

where $0 \leq \eta \leq 1$ denotes the producer's bargaining power and $V_{p}^{d}$ and $V_{w}$ are taken as given. Because production and exchange sectors are distinct sectors, there is no general reason to assume that the producer's bargaining power is equal to the seller's bargaining power. As a consequence, we allow for $\eta \neq \sigma$. 
The first order condition for the unconstrained version of problem (29) is

$$
q_{c}=\eta+\eta V_{w}-(1-\eta) V_{p}^{d}-\eta \delta
$$

where

$$
V_{w}=\frac{1-Q_{m}^{*}(\sigma)-(r+1) \delta}{r(r+2)} \text { and } V_{p}^{d}=\frac{Q_{c}}{r}
$$

Therefore, we can rewrite the first order condition as follows

$$
q_{c}=\eta+\eta\left[\frac{1-Q_{m}^{*}(\sigma)-(r+1) \delta}{r(r+2)}\right]-\frac{(1-\eta) Q_{c}}{r}-\eta \delta=G\left(Q_{m}^{*}(\sigma), Q_{c}\right)
$$

To identify the rational expectation equilibrium value $Q_{c}^{*}$ we have to look for a fixed point for (31). However, in this case, we have to distinguish between constrained and unconstrained solutions.

Lemma 2 For every $\eta \in[0,1)$ a value $0<\sigma_{\eta}<\bar{\sigma}$ always exists such that: (i) if $\sigma_{\eta} \leq \sigma \leq 1$ there is always a unique value $0 \leq Q_{c}^{*} \leq 1-\delta$ such that $Q_{c}^{*}=G\left(Q_{m}^{*}(\sigma), Q_{c}^{*}\right)$; (ii) if $0 \leq \sigma<\sigma_{\eta}$ the rational expectation solution for problem (29) is constrained and $Q_{c}^{*}=1-\delta$

Proof. First of all, we observe that $G\left(Q_{m}^{*}(\sigma), Q_{c}\right)$ is a continuous function with $\partial G / \partial Q_{c}<0$ and $G\left(Q_{m}^{*}(\sigma), 0\right)>0$. It follows that for a given value $Q_{m}^{*}(\sigma)$ we have $\min \left\{G\left(Q_{m}^{*}(\sigma), Q_{c}\right)\right\}=G\left(Q_{m}^{*}(\sigma), 1-\delta\right)$. Consider now $G\left(Q_{m}^{*}(\bar{\sigma}), 1-\delta\right)$ and suppose that $\sigma \geq \bar{\sigma}$. Hence

$$
G\left(Q_{m}^{*}(\sigma), 1-\delta\right)=G\left(Q_{m}^{*}(\bar{\sigma}), 1-\delta\right)=(1-\delta)\left[\frac{\eta(r+1)-1}{r}\right]<1-\delta
$$

However, if $G\left(Q_{m}^{*}(\bar{\sigma}), 1-\delta\right)<1-\delta$, by continuity there is always a unique value $\sigma_{\eta}<\bar{\sigma}$ such that if $\sigma \geq \sigma_{\eta}, G\left(Q_{m}^{*}(\sigma), 1-\delta\right)<1-\delta$; but this means that, under the previous restriction on $\sigma$, there is always a unique value $0 \leq Q_{c}^{*} \leq 1-\delta$ such that $Q_{c}^{*}=G\left(Q_{m}^{*}(\sigma), Q_{c}^{*}\right)$. Point (ii) is straightforward.

The unconstrained solution for (29) can be obtained from (31) and gives

$$
Q_{c}^{*}=\eta\left[\frac{(r+1)\left[(r+1)^{2}(1-\delta)-\delta r\right]-\sigma[r+1-\delta]}{(r+2)(r+1-\eta)(r+1)}\right]
$$

The following proposition states that the constrained solution can not be consistent with a sustainable monetary equilibrium. This happens because $Q_{c}^{*}$ is too high with respect to $Q_{m}^{*}(\sigma)$.

Proposition 9 If the solution for problem (29) is constrained, a sustainable monetary equilibrium for the generalized economy never exists. 
Proof. As we know, the solution for problem (29) is constrained if and only if $0 \leq \sigma<$ $\sigma_{\eta}$. Under this circumstance, the sustainability condition is

$$
\frac{Q_{m}^{*}(\sigma)}{r+2}>1-\delta \Leftrightarrow \sigma>\frac{(1-\delta)(r+1)(r+2)}{r+1-\delta}=\widetilde{\sigma}
$$

It is immediate to verify that $\widetilde{\sigma}>\bar{\sigma}$. However $\sigma_{\eta}<\bar{\sigma} \Rightarrow \sigma<\widetilde{\sigma}$ and the sustainability condition can be never satisfied.

Hence, in order to look for a sustainable monetary equilibrium we can restrict our attention to the unconstrained solutions set.

Proposition 10 For every $0 \leq \delta \leq 1 /(1+r), r>0$ and $0 \leq \eta \leq h(\delta, r)$, a sustainable monetary equilibrium for the generalized economy always exists if and only if $\sigma \geq \eta H(r, \delta)$ where $H(r, \delta)$ and $h(\delta, r)$ are decreasing functions of $\delta$.

Proof. From Proposition (9) we need $\sigma \geq \sigma_{\eta}$. By equation (32), the sustainability condition $Q_{m}^{*} /(r+2) \geq Q_{c}^{*}$ can be written in the following way

$$
\sigma \geq \eta\left[\frac{(r+1)^{2}(1-\delta)-r \delta}{(r+1-\delta)}\right]=\eta H(\delta)
$$

It is quite easy to verify that $\partial H(\delta) / \partial \delta<0$; moreover, it is possible to prove that $\eta H(\delta) \geq \sigma_{\eta}$ for every admissible $\eta$ ( (see appendix A.2) so that our initial assumption holds true. However, the previous condition does not prove that a sustainable monetary equilibrium exists for a non empty parameters space, that is $\eta H(r, \delta) \leq \bar{\sigma}$. In other words, we have to verify that the inequality $\eta H(r, \delta) \leq \bar{\sigma}$ holds true for a significant restriction. After some algebra we obtain $\eta H(r, \delta) \leq \bar{\sigma} \Leftrightarrow$

$$
\eta \leq \frac{(1+r)-\delta(r+1)^{2}}{(1+r)-\delta(r+1)^{2}+r(r+1-\delta)}=h(r, \delta)
$$

where $\partial h / \partial \delta<0$ and $h(r, \delta) \geq 0$.

The first thing that we have to note is that while in a non-generalized full-employment economy the assumption $\delta=0$ destroys the monetary equilibrium, in a generalized economy a sustainable monetary equilibrium exists even if we assume a zero labour disutility.

Proposition 11 Suppose that $\delta=0$ and $r>0$. Then a sustainable monetary equilibrium exists if and only if $\eta \in[0,1 /(1+r)]$ and $\sigma \geq(1+r) \eta$

Proof. It follows immediately from Proposition 10

As we have seen, if $\delta=0$, the labour disutility effect becomes irrelevant. However, in presence of heterogeneous bargaining power, the monetary contract can be still robust to a co-operative deviation. This last result is entirely due to the fact that a monetary 
arrangement involves not only a different bargain timing, but also different sectors with respect to a co-operative economy. Even if producers can not take advantage from the first difference, they can still take advantage from the second one, provided that their bargaining power in the exchange sector is sufficiently high relative to their bargaining power in the production sector.

More in general, the effect due to the existence of heterogeneous bargaining weights $a d d s$ to the monetary illusion effect. This makes the monetary equilibrium more robust and, at the same time, implies that the two effects are substitute. From Proposition 10 we know that $H(r, \delta)$ is a decreasing function of $\delta$. If we strengthen the labour disutility effect, the threshold value for $\sigma$ decreases and the monetary equilibrium can be consistent with a seller's bargaining power lower than before. In particular, we observe that if $\delta>(r+1) /(r+3), H(r, \delta)<1$ and this implies that a monetary equilibrium could exist even if the producers' bargaining power in the exchange sector is lower than their bargaining power in the production sector.

\section{$4 \quad$ Efficiency}

In the previous sections we proved that, under interpretable parameters restrictions, a monetary equilibrium is sustainable. This means that if we start from a monetary equilibrium no producer has incentive to deviate from the monetary contractual arrangement. However we may ask if the entrepreneurs could prefer a monetary contract also from an ex ante perspective.

The monetary equilibrium is, undoubtedly, inefficient because it implies a longer chain of transactions without any social advantage. As a matter of fact, production can not be increased and the monetary contract reduces the dimension of the production sector.

However, even if the monetary equilibrium can not be a first best, it can be still $e x$ ante optimal for the entrepreneurs. In our framework, the contractual arrangement is an entrepreneurs' prerogative and, from their point of view, the monetary contract can be preferred because they can take advantage from surplus distribution. The question is whether the inefficiency loss may be compensated by the gain from trade earned by the entrepreneurs or not. In order to explore this question we have to compare the producer's value function in a co-operative economy to their corresponding value function in a monetary economy.

The optimality conditions in a co-operative economy simplifies to the following couple of Bellmann equations.

$$
\begin{gathered}
r V_{p}^{c}=Q_{c e} \\
r V_{w}^{c}=1-Q_{c e}-\delta
\end{gathered}
$$

where $Q_{c p}$ is determined as a fixed point of the following best reply function (see equation (30))

$$
q_{c}=\eta+\eta \frac{\left(1-Q_{c}-\delta\right)}{r}-(1-\eta) \frac{Q_{c}}{r}-\eta \delta
$$


If $\eta \in[0,1 /(1+r)]$, there is always a stationary solution for the previous equation and, in particular, we obtain $Q_{c e}^{*}=(1-\delta) \eta$.

The monetary equilibrium will be preferred ex-ante to the co-operative one if and only if $V_{p} \geq V_{p}^{c}$, i.e. $Q_{m}^{*}(\sigma) /(r+2) \geq(1-\delta) \eta$. From equation (28) we obtain the following condition:

$$
\sigma \geq\left[\frac{(1-\delta)(r+1)(r+2)}{r+1-\delta}\right] \eta=B(r, \delta) \eta
$$

We conclude that if the sellers' bargaining power is sufficiently high relative to the producers' bargaining power, the inefficiency loss implied by a monetary contract will be more than compensated by the gain from surplus distribution. Moreover, we observe that $B(r, \delta) \geq H(r, \delta)$; in order to obtain an ex-ante optimal monetary equilibrium we have to increase the threshold value for $\sigma$. At the same time, $\partial B(r, \delta) / \partial \delta<0$; the labour disutility effect allow us to weaken the restriction on $\sigma$. In particular we have $\min \{B(r, \delta)\}=(1+r)$ so that, even in the most favorable case, we need $\sigma>\eta$.

This last result clarifies one point. The labour disutility effect plays a major role in an ex post perspective, that is once we are placed in a monetary equilibrium. In this case the producers who offer a monetary contract have a competitive advantage with respect to a deviating producer who offers a co-operative contract, simply because this last one bargains over $q$ with active workers having the same expected utility but also a positive labour disutility cost. On the contrary, if we look at an ex ante perspective, the monetary illusion effect ceases to be crucial, at least as an absolute argument in favour of the monetary contract, and the heterogeneous bargaining powers effect comes to the fore.

\section{Conclusions}

We studied a search theoretic model where agents are heterogeneous with respect to the role played in the economic system. Entrepreneurs hold a dominant role, as they have an exclusive access to the credit system and can decide about the transaction strategy used to carry out production. On the contrary, workers play a residual role as they can only decide whether to participate or not to production and exchange activity. This appears to be a substantial departure from the standard search theoretic framework, insofar as the monetary solution is driven essentially by the objective function of a single class of agents.

In absence of a double coincidence of wants problem, the co-operative technology dominates the monetary transaction technology unambiguously. This happens because: (a) the single production levels are constant by assumption, (b) money wastes time involving a longer transaction chain. Nevertheless, the surplus distribution depends on transaction technology and this is a crucial argument for the existence of a monetary equilibrium. Money is created and destroyed, de facto, by the entrepreneurs, whereas the bank act only as a policeman to avoid opportunistic behavior. As a consequence, in order to look for a rationale for money we have to look at entrepreneurs' incentives. If 
entrepreneurs can take a large enough advantage from a monetary transaction technology, without extracting all the surplus from workers, the monetary equilibrium becomes a credible outcome.

In particular we identified two factors which explain the existence of a monetary equilibrium: the labour disutility cost and the relative seller's bargaining power. An increase in the labour disutility reduces the entrepreneurs' output share in a monetary as well as in a co-operative economy. At the same time it makes less plausible a deviation from the monetary contract. This happens because of the labour disutility effect which causes a relative increase in the real wage to be paid in a deviating co-operative contract.

The relative bargaining power of the seller has a direct positive effect on the output share of the entrepreneurs. There are two threshold levels for the relative bargaining power of the seller, $H(r, \delta)$ and $B(r, \delta)$. If the seller's bargaining power is larger that the first one but less than the second one, we can state that the monetary equilibrium is robust to co-operative deviations. If it is higher that the second one, entrepreneurs would prefer the monetary equilibrium also from an ex ante perspective. More precisely, the efficiency loss due to the extension of the transaction chain will be more than compensated by the gain in term of output share. Moreover, the monetary illusion effect and the relative bargaining power effect are substitute. An increase in the labour disutility costs reduces both of the threshold values $H(r, \delta)$ and $B(r, \delta)$.

\section{Appendix}

\section{The relationship between $\eta H(\delta)$ and $\sigma_{\eta}$}

We have to prove that $\sigma_{\eta} \leq \eta H(\delta)$ for every admissible $\eta$. The value $\sigma_{\eta}$ can be obtained imposing the condition $G\left(Q_{m}^{*}(\sigma), 1-\delta\right)=1-\delta$ and solving for $\sigma$. After some algebra we obtain

$$
\sigma_{\eta}=\frac{(r+1)[1-\delta(r+1)]}{r+1-\delta}-\left(\frac{1-\eta}{\eta}\right)\left[\frac{(1-\delta)(1+r)^{2}(r+2)}{r+1-\delta}\right]=(*)
$$

Note that $\sigma_{\eta}$ is an increasing continuous function of $\eta$ and if $\eta=0, \sigma_{\eta}<0$ while if $\eta=1, \sigma_{\eta}>0$. This means that a value $\eta^{*}$ exists such that $\sigma_{\eta}=0$ if $\eta=\eta^{*}, \sigma_{\eta}<0$ if $\eta<\eta^{*}$, and $\sigma_{\eta}>0$ if $\eta \geq \eta^{*}$. The function $\sigma_{\eta}$ can be now redefined in the following way

$$
\sigma_{\eta}=\left\{\begin{array}{cl}
(*) & \text { if } \eta \geq \eta^{*} \\
0 & \text { if } \eta<\eta^{*}
\end{array}\right.
$$

The value $\eta H(\delta)$ is given by

$$
\eta H(\delta)=\eta\left[\frac{(r+1)^{2}(1-\delta)-r \delta}{(r+1-\delta)}\right]
$$

Let us now define the function $\Delta(\eta)=\eta H(\delta)-\sigma_{\eta}$. It is immediate to verify that $\Delta(\eta) \geq 0$ for $\eta \in\left[0, \eta^{*}\right), \Delta\left(\eta^{*}\right)>0$, and $\Delta(1) \geq 0$ for every $0 \leq \delta \leq 1 /(1+r)$. 
Moreover

$$
\frac{\partial \Delta(\eta)}{\partial \eta}<0 \text { for every } \eta \in\left[\eta^{*}, 1\right]
$$

It follows that $\Delta(\eta) \geq 0$ for every admissible $\eta$ and this means that the inequality $\sigma_{\eta} \leq \eta H(\delta)$ is always satisfied.

\section{References}

[1] Amendola, N. (2008), A Selection Mechanism for the Barter equilibrium in the Search Theoretic Monetary Model, Economics Bulletin, Vol. 5, no 2, pp.1-10

[2] Berentsen, A. and Rocheteau, G. (2003), Money and the Gains from Trade, International Economic Review, Vol. 44, No 1

[3] Binmore, K., Rubinstein, A., Wolinsky, A. (1986), The Nash Bargaining Solution in Economic Modelling, RAND Journal of Economics, Vol. 17, No 2, pp. 176-188.

[4] Corabae, D.,Temzelides, T. and Wright, R. (2002), Matching and Money, American Economic Review, Papers and Proceedings, 92, pp. 67-71.

[5] Corabae, D.,Temzelides, T. and Wright, R. (2003), Direct Matching and Monetary Exchange, Econometrica, Vol. 71, No 3, pp. 731-756.

[6] Engineer, M., Shi, S. (1998), Asymmetry, imperfectly transferable utility, and the role of fiat money in improving terms of trade, Journal of Monetary Economics, 41, pp. 153-183.

[7] Engineer, M., Shi, S. (2001), Bargains, Barter, and Money, Review of Economic Dynamics, 4, 188-209.

[8] Keynes, J.M. (1933) The distinction between a co-operative economy and an entrepreneur economy, The collected writings of John Maynard Keynes, vol. XXIX, London, Macmillan, 1979

[9] Kiyotaki, N. and Wright, R. (1991) A Contribution to the Pure Theory of Money, Journal of Economic Theory, 53, 215-235

[10] Kiyotaki, N. and Wright, R. (1993) A Search Theoretic Approach to Monetary Economics, American Economic Review, 83, 63-77

[11] Rupert, P., Shindler, M., Shevchenko, A., Wright, R. (2000), The Search-Theoretic Approach to Monetary Economics: A Primer, Federal Reserve Bank of Cleveland Economic Review, v.36, iss.4, 10-28

[12] Shi, S. (2006), Viewpoint: A Microfoundation of Monetary Economics, Canadian Journal of Economics, 39, 643-688 
[13] Trejos, A. and Wright, R. (1995), Search, Bargaining, Money and Prices, Journal of Political Economy, vol. 103, n.1 